\title{
International
}

\section{THE EFFECTIVENESS OF BALANCE TRAINING WITHOUT VISUAL FEEDBACK IN STROKE SUBJECTS}

Search engine: www.ijmaes.org

\section{AMJAD ANNETHATTIL ${ }^{1}$, JIBI PAUL ${ }^{2}$, PRAVEEN KUMAR ${ }^{2}$, RAMANATHAN ${ }^{2}$, ABHISHEK KUMAR}

\section{Authors:}

${ }^{2}$ Faculty of Physiotherapy, Dr. MGR. Educational and Research Institute, Chennai, Tamilnadu, India.

${ }^{3}$ Lecturer in Physiotherapy, Florence College of Physiotherapy, Bangalore, Karnataka, India

\section{Corresponding Author:}

${ }^{1}$ Amjad Annethattil, Stroke care Association, Physiotherapy Specialist, Assam down town University, India. E Mail: amjus2001@yahoo.co.in

\section{Abstract}

Background of the study:The dramatic increase in the incidence and prevalence of neurological disorders like Stroke has always demanded the need of new interventions in limiting the disability outcome following stroke. Several research work have been conducted with the process still continuing towards limiting the disability till its controlled fully. Aims and Objectives: This study coducted to find the effectiveness of balance training with deprivation of visual feedback in stroke subjects. Methodology:Total number of fifteen subjects participated in this study. The duration of the study was four continuous weeks, with five sittings of sixty minutes each per week. Data was collected using Timed Up and Go test and Berg balance scale. The statistical analysis used for the study was paired Ttest and Wilcoxons signed rank test. Result: Subjects with masked vision showed better outcomes on berg balance scale and Timed Up and Go test with $P<0.001$. Conclusion: Balance performance and movement can be improved with deprived vision training on stroke subjects.

Key words: Stroke, Deprivation of Visual feedback, Balance, Berg balance scale, Timed Up and Go test.

Received on $09^{\text {th }}$ Feb 2018, Revised $21^{\text {th }}$ Feb 2018, Accepted on $26^{\text {th }}$ Feb 2018 


\section{INTRODUCTION}

Stroke patients typically demonstrate asymmetry with most of the weight bearing in sitting or standing shifted to non paretic side, at the same time they even demonstrate increased postural sway in standing and delays in onset of motor activity, abnormal timing, sequencing of muscle activity and abnormal co-contraction results in the disorganization of the postural symmetry. The typical response for compensating the proximal muscles activation prior to the distal muscles includes excessive hip and knee movements on the contrary corrective response to perturbations or destabilizing forces are frequently inadequate and results in loss of balance, with patients typically falling in the direction of weakness.

Visual field deficits like homonymous hemianopsia occur with lesion involving the optic radiation in internal capsule of middle cerebral artery distribution or primary visual cortex of posterior cerebral artery distribution occurring in twenty-six percent of patients with stroke ${ }^{1}$.

This excessive reliance on vision is reversible after a vision deprived balance therapy by inducing subject to use somatic sensory and vestibular inputs to rely less on visual inputs wherein proprioception has been found to improve balance in stroke subjects ${ }^{2,3}$.

Sensory motor training emphasizes postural control and progressive challenges to the somatosensory system to restore normal motor programmes in patients with chronic musculoskeletal pain. And hence stated sensory motor training as a global approach towards balance training 4 .

There are many training methods to improve flexibility and function among stroke patients 5,6 . The predominant influence of visual input constitutesnotural compensatory strategy for coping with initial imbalance and that traditional rehabilitation reinforces this excessive visual reliance by focusing on visual compensation rather than restoring the normal use of sensory inputs ${ }^{7,8}$.
It is important to identify effective stroke rehabilitation strategies as the number of stroke survivors and medical cost increases. Clinical test for sensory interaction in balance is a simple inexpensive test that can be used to test sensory organisation. ${ }^{9,10}$

A quick measure of basic mobility and balance has developed which provided a reliable screening including an assessment of level walking through the timed get up and go test with established reliability, validity and response to contribute to the measure of balance $^{11}$.

\section{METHODOLOGY}

Study design: This was an experimental study with pre post test analysis.

Setting of the study: Stroke patients referred to the out patient department of physiotherapy at Florence rehabilitation center, Bangalore.

Sampling techniques: Those subjects diagnosed as having stroke were selected as study Subjects, recruited by simple random sampling in rehabilitation department with hemiplegia caused by first and only cerebral hemispheric stroke. Informed consent received from all the subjects participated in the study.

Fifteen subjects were selected randomly from thirty subjects with twelve males and three females.

Inclusion Criteria: Patients diagnosed with stroke and were able to walk independently without human support with age group of 4565 years. They were with single attack stroke subjects with a duration of more than six months and less than one year six months and grade 2 and above by modified Asworth spasticity scale, the muscle power of 3 and above on manual muscle testing.

Exclusion Criteria: Subjects with perceptual and cognitive deficits.Subjects with any lower limb joint pathologies like arthritis, limb length discrepancies.Patients with visual impairments and Visio-spatial disorders. 
Measurement tools: Berg balance scale and Timed Up and Go test.

Materials used: Exercise mat, Treadmill, Static bicycle, Stop watch.

Procedure:Fifteen subjects were administered conventional therapy with deprived vision. An initial reading of the total berg balance scale and Timed Up and Go test taken before starting the intervention and final data collected at end of fourth week of the training.

\section{Intervention}

Mobility exercise: Active, active assisted or passive as required pertaining to the individual joint ranges and performed ten repetitions, spinal mobility exercise were also induced apart from the exercises for joints of upper extremity and lower extremity.
Treatment started with five minutes of cycling on a static bicycle.

Strengthening Exercises: Resistance training was typically accomplished for the weaker group of muscles pertaining to their grades from manual muscle testing, they were induced with Resisted exercises for all individual muscle groups with the help of optimal resistance weigh cuffs throughout the range of motion commands were given for completing the range and positioning of the extremities. All exercise performed ten repetitions.

Balance Exercises: Balance training was given through various positions in mat. Exercises performed in sitting progressed to balance training in standing. Activities in mat included bridging, weight shifting trainining from one side to the other in standing, bridging with single limb support, weight shifts in sitting, quadriped position all exercise performed ten repetitions.

\section{RESULT}

\section{Timed Up And Go Test:}

\begin{tabular}{|c|c|c|c|c|c|c|}
\hline $\begin{array}{c}\text { Get up and } \\
\text { Go Test }\end{array}$ & \multicolumn{2}{|c|}{ Pre } & \multicolumn{2}{c|}{ Post } & \multirow{2}{*}{ T-value } & \multirow{2}{*}{ P-value } \\
\hline \multirow{2}{*}{ Group A } & Mean & S.D & Mean & S.D & & \\
\cline { 2 - 6 } & 14.867 & 1.727 & 11.000 & 0.926 & 12.61 & $0.000^{* \cdots}$ \\
\hline
\end{tabular}

Table 1: Intra group analysis of Timed Up and Go test

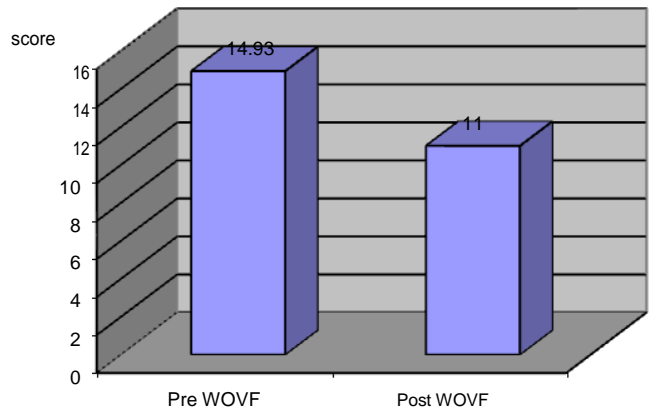

Graph 2: Pre and Post Timed Up and Go test without visual feedback(WOVF)
Table 1 shows the pre treatment mean value of group A in Timed get up and go as 14.933 with a Standard deviation of \pm 2.51 , the post treatment mean of 11.000 and Standard deviation of \pm 0.926 The $t$-value of 8.50 and the $P$-value as $0.000(p<0.05)$ Here the difference of mean outcome with a $t$ value of 8.50 is statistically significant. 


\section{Berg balance scale:}

Intra group analysis was done using Wilcoxons signed rank test. The data present in table 2 shows the mean rank as 8 , positive sum of ranks as 120 and negative sum of ranks as 0 ,With the critical value of 8 and the $P$ - value as $0.000(p<0.05)$ Group-A shows a statistically significant outcomes for balance.

\begin{tabular}{|c|c|c|c|c|}
\hline Berg balance scale & Mean rank & Sum of ranks & Critical value & P-value \\
\hline Group-A & 8 & $0-120.0$ & 30 & $0.000^{*}$ \\
\hline
\end{tabular}

Table 2: Intra Group analysis of Berg balance scale

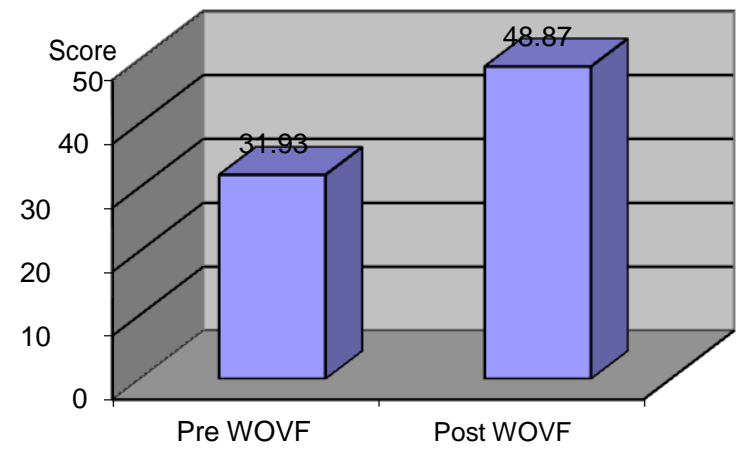

Graph 2: Pre and Post Berg balance scale without visual feedback(WOVF)

\section{DISCUSSION}

Stroke has been found to impair balance. In our study we have tried to find out the individual effects of conventional physiotherapy with masked vision on balance of the patient.

Parameters named as Berg balance scale with a fourteen phase five point ordinal scale score, Modified Ashworth scale and the Timed Get up and go test with a timed performance scoring. The present study suggests that the vision deprived balance training improves the balance parameters significantly $(p<0.05)$ as $p=0.000$. On Intra group analysis vision masked balance exercise and conventional

physiotherapy was effective in improving the balance in hemeperetic patients following stroke, Supported by Isabelle who stated that vision overuse may be a compensatory strategy for coping with initial imbalance exacerbated by traditional rehabilitation, hence vision deprived rehabilitation improves balance more effectively.

Mars den studied the vestibular control of balance after stroke by examing the vestibular control of balance following stroke. Walker C Studied the use of visual feedback in retraining balance following acute stroke and concluded that balance training along with visual feedback in addition to regular therapy affords no added benefits when offered in early stages of rehabilitation following stroke.The rehabilitation programme includes relaxation exercises; mobilization exercises, strengthening exercise and balance exercise in lying, sitting on mat and on standing ${ }^{12,13}$.

Proprioception and somatosensory pathway has been found to contribute to the vision deprived therapy to a great extent. Masked vision enhances concentration through the somatosensory pathway towards promotion of balance through sensory reeducation ${ }^{14,15}$.

This study tries to find out the effects of vision deprived balance therapy in the form of masked vision along with conventional therapy on reestablishing balance in stroke subjects. Conventional therapy brings about improvement in balance, functional mobility and activities of daily living. Stroke patients are disabled by the weakness persisting after stroke, deprived visual feedback promotes the use of affected side and prevents the compensatory over use adaptability ${ }^{16,17,18}$. 


\section{CONCLUSION}

Impaired balance has been found to be one of the common disabling factor following stroke, influencing the activities of daily living.

The promotion and restoration of balance and independent functional mobility has always been demanding in subjects suffering from stroke.The results showed that the conventional therapy along with masked vision could bring about significant changes in balance, mobility and function of patients suffering from balance impairment post stroke.

\section{REFERENCES}

1. Dettmann M.A; Linder M.T; Sepic S.B; Relationship among walking performance, postural stability and functional assessment of the hemi plegic patient Am.J.Phys.Med, 1987; 66 (2): 77-90.

2. Walker C, Bruwer B.J, Culham E.G, Use of visual feedback in retraining balance following acute stroke; Phys.ther, 2000; 80(9): 886-895.

3. Bonanl.V, F.M Colle, J.P.Guichard et.al, Reliance on visual information after stroke Part-I Balance on dynamic posturography; Arch Phys Med Rehab, 2004;85:268-273.

4. Phil Page; Somatosensory training a global approach for balance training Bodywork and movement therapies; 2005;75-79.

5. Jibi Paul, Janaki S (2017). Effectiveness of crossed reciprocal inhibition to reduce biceps brachii spasticity in stroke subjectsa random controlled trial, IJMAES; 3 (1): pp250-259.

6. Jibi Paul (2014). Comparative study of combined effect of task oriented motor relearning program and thermal stimulation over upper limb motor function among stroke subjects. IJPHY; Vol.1 (4): pp227-232.

7. J F Marsden, D E Playbrol and B L day; The vestibular control of balance after stroke Neurology neurosurgery and psychiatry; 2005;76: 670-679.
8. Cohen, H.et.al; A study of Clinical test of sensory interaction in balance; PhysTher; 1993; 73: 346.

9. Nashner, L; Adaptive reflexes controlling human posture; Exp Brain, 1976; 26:59.

10. Ellen A Limpkin and Diana M Bautista; Feeling the pressure I mammalian somatosensation; Neurobio, 2005; 15: 382-388.

11. Mathias, S, et.al; Balance in elderly patient, The Get up and Go Test; Arch Phys Med Rehabil, 1986; 67:387.

12. Isabelle $V$ et.al; Reliance on visual information after stroke Part-I Balance on dynamic posturography; Arch Phys Med Rehabil, 2004; 85(2): 268-273.

13. Isabelle $V$ et.al; Reliance on visual information after stroke Part-II Archive of phys Med Rehabil, 2004; 85: 274-278.

14. Berg et.al; The Balance scale reliability assessment for elderly residents and patients with an acute stroke; Scand J Rehabil Med, 1995; 27:27.

15. Laura Adomatis et.al; An intensive massed practice approach to retraining balance post-stroke; Gait and Posture, 2005; 22 (2): 154-163.

16. R.A Geiger, J.B.Allen et.al; Balance and mobility following stroke; Effects of physical therapy intervention with and without biofeedback or forceplate training; PhysTher 2001; 81: 995-1005.

17. C.Walker, B.J.Brouwen and E.G Culham; Use of visual feedback in retraining balance following acute stroke; PhysTher 2000; 80: 886-895.

18. Jibi Paul, Pradeep Balakrishnan, Mohd Izham (2014). Comparative effect of vision deprived balance training over free vision balance training in stroke subjects. IJPHY; Vol.1 (2): pp 46-53.

\section{Citation:}

Amjad Annethattil, Jibi Paul, Praveen Kumar, Ramanathan K, Abhishek Kumar.The effectiveness of balance training without visual feedback in stroke subjects, IJMAES, 2018; 4 (1), 448-452. 